\title{
The normal distribution in scaling subjective stimulus differences: Less "normal" than we think?
}

\author{
ÅKE HELLSTRÖM \\ Stockholm University, Stockholm, Sweden
}

\begin{abstract}
The Gaussian, or "normal," distribution is routinely used to model distributions of subjective quantities. This practice rests on a trust in the central limit theorem, yet this theorem does not cover the case of a mixture of Gaussian distributions with different standard deviations, which yields a distribution that is heavier tailed than the Gaussian. In psychophysical judgment experiments, this may result, for example, from fluctuating or interindividually varying attention. Two candidates for describing this commonly encountered type of distribution are the logistic distribution and the $t$ distribution with a small number of degrees of freedom. In reanalyses of experimental data on three-category loudness comparisons, as well as in a Monte Carlo simulation, $t(4)$ was found to model the underlying mixed inter- and intraindividual distribution of subjective loudness differences quite satisfactorily.
\end{abstract}

According to the central limit theorem, the distribution of a sum (or mean) of a large number of stochastic variables approaches the Gaussian, or normal, distribution (ND) under a wide range of conditions (see Cramér, 1946, pp. 213ff). For an ND variable $X$, its deviate $z=$ $\left(x-\mu_{x}\right) / \sigma_{x}$, with mean 0 and standard deviation $(S D) 1$, has the density function $\phi(z)=(2 \pi)^{-1 / 2} e^{-1 / 2 z^{2}}$. The ND has long been used to model various physical and biological processes. In psychology, it is the cornerstone of the statistics taught to beginning students, and it is traditionally assumed to hold not only for intelligence (Galton, 1869; see Boring, 1950, chap. 10), but also (over observers as well as trials) for the subjective quantities that are judged, categorized, or compared in psychophysical tasks (Thurstone, 1927a, 1927b). Its ogival distribution function is commonly assumed (the phi-gamma hypothesis; Guilford, 1954) to adequately describe, for instance, the psychometric function in an experiment with the method of constant stimulus differences-that is, the proportion of judgments implying that the comparison stimulus is heavier than the standard as a function of the magnitude of the former. Transforming the judgment proportions to standard normal deviates ( $z$ values) is a routine procedure for linearizing psychometric function plots, as well as, for example, receiver-operating characteristic curves from signal detection experiments. One major innovation is the use of the lognormal distribution, on the assumption that the

This research was supported by the Swedish Council for Research in the Humanities and Social Sciences. I thank John Öhrvik, Department of Statistics, Stockholm University, for valuable comments and suggestions, including that of using the $t$ distribution. Correspondence should be addressed to $\dot{A}$. Hellström, Department of Psychology, Stockholm University, S-106 91 Stockholm, Sweden.
ND holds for the logarithm of the subjective quantity, not for the quantity itself (the phi-log gamma hypothesis, Guilford, 1954; see also Eisler, 1965).

However, in many instances, neither the ND nor the lognormal distribution adequately describes the distribution of the subjective quantity under study. In fact, human judgments are no exception to the following general observation of Tukey and McLaughlin (1963):

\begin{abstract}
The Gaussian or Laplacian distribution, to the physicist the Maxwellian distribution, has long been known to the statistician as the normal distribution. However little noticed such a commonly-used name becomes, shades of its original meaning continue to cling-distributions that are not normal are, by at least slight implication, pathological .... In a well-developed area such an attitude can only be respectable if it reflects the facts-if the usual is at least close to the "normal" in behaviour. This is not the case in almost all of the instances of data analysis [with] which the writers-and, they believe, most practising statisticians, have come in contact. The typical distribution of errors and fluctuations has a shape whose tails are longer than that of a Gaussian distribution. (pp. 331-332)
\end{abstract}

Consequently, the frequency of notably deviant values (outliers) is typically higher than would be expected if the ND were the true distribution. "Robust" measures of central tendency and variability that are less affected by outliers than are the arithmetic mean and $S D$ have been developed (see Hoaglin, Mosteller, \& Tukey, 1983), and these deserve increased attention in, for instance, psychophysical scaling.

In psychophysical experiments as well as in everyday judgments, one factor that may cause the long-tail phenomenon consists of fluctuation in the judge's attention level, with occasional low attention levels leading to increased judgment variability. The judgments can then be 
viewed as if they were sampled from several populations with different variabilities, those with high variability being the minority. It is important to realize that this does not mean that one averages random variables, but instead that one mixes distributions. Even if each judgment population has an ND, the total judgment distribution will be heavier tailed than the ND.

In this article, I shall present empirical evidence for the adequacy of this analysis of the problem in a particular case of psychophysical scaling, and I shall also suggest remedies. I shall begin by discussing some alternative distribution assumptions.

One approach to modeling the shape of a mixture of NDs involves the contaminated normal distribution (CND; see Hoaglin et al., 1983, chap. 10). The CND is based on the assumption that each observation is taken with probability $1-\alpha$ from a standard ND with mean 0 and $S D 1$, and with probability $\alpha<.5$ (say, .1) from another ND with mean 0 and $S D K>1$ (say, 3). The distribution function for the ND being $\Phi(x)$, that of the CND (denoted by $\mathrm{CN}(\alpha: K)$ is $F(x)=(1-\alpha) \Phi(x)+$ $\alpha \Phi(x / K)$. It follows that, in comparison with the standard $\mathrm{ND}$, the CND, rescaled to restore the $S D$ to 1 from its resulting value of $\left[\alpha \cdot 1^{2}+(1-\alpha) \cdot K^{2}\right]^{1 / 2}$, is more heavytailed. The CND is a simplified model that should work best when a few observations are "wild" and the rest approach an ND. In other cases, it may be more realistic to assume a mixture of more than two NDs with different $S D$ s (those with large $S D$ s being the minority, so that the $S D$ distribution is positively skewed).

One distribution that may serve as a useful model in the last-mentioned type of case is the $t$ distribution, which is the distribution of the product $t(d f)=U \cdot V$, where $U$ and $V$ are independent random variables, $U$ being ND with mean 0 and $S D 1$, and $V$ distributed as $\left[\chi^{2}(d f) / d f\right]$, where $d f$ is the number of degrees of freedom. The smaller the $d f$, the more positively skewed the distribution of $V$ and the larger its $S D$. This definition of the $t$ distribution suggests that $t$ with small $d f$ might be useful as a model for subjective quantities distributed as mixtures of NDs whose $S D$ s vary similarly to those of $V$ (which seems quite plausible, e.g., in situations with fluctuating attention). The density function of $t(d f)$ is $f_{t}(d f)( \pm x)=\{\Gamma[(d f+1) / 2]\} /$ $\left[(\pi d f)^{1 / 2} \Gamma(d f / 2)\right]\left(1+x^{2} / d f\right)^{-1 / 2(d f+1)}$, where $\Gamma(n)$ is the gamma function, which for integer values of $n$ is equal to $n !$. $t$ is heavier tailed than the ND, becomes more so the fewer the $d f$, and approaches the ND as $d f \rightarrow \infty$. For $d f>2$, the $S D$ of $t$ equals $[d f /(d f-2)]^{1 / 2}$; hence dividing the $t$ values by this value rescales them to an $S D$ of 1 , making them commensurable with $z$.

The logistic transform is similar to the cumulative ND (slightly longer tailed), but easier to calculate (Bush, 1963). It is used in bioassay (Berkson, 1944, 1953), as well as in item difficulty scaling in test psychology (Rasch, 1960 ) and in logistic regression models for binary variables in general (e.g., Feinberg, 1980, chap. 6). Luce's choice axiom (Luce, 1959; Luce \& Galanter, 1963) predicts that in two-category stimulus comparison the proportion $p$ of judgments implying that the comparison stimulus $>$ standard can be described by the logistic transform, $p=\lambda(\ell)=\left(1+e^{-\ell}\right)^{-1}$ [where the "logit" $\ell=(x-b) / \mathrm{a} ; x$ is the magnitude of the comparison stimulus; $a$ and $b$ are fitted constants], or $\ell=\ln [p /(1-p)]$. For this case, the logistic transform is often considered the main alternative to the cumulative ND with some empirical support (Burke \& Zinnes, 1965) and with Luce's theoretical foundation.

$\lambda(\ell)$ is also the distribution function of the logistic distribution, whose density function is most conveniently expressed as

$$
f\left(z_{\ell}\right)=(\pi / \sqrt{ } 3) e^{-\pi z \ell / \sqrt{ } 3 /\left(1+e^{-\pi z \ell / \sqrt{ } 3}\right)^{2},}
$$

where $z_{\ell}=\left(\ell-\mu_{\ell}\right) / \sigma_{\ell}$ (Johnson \& Kotz, 1970, chap. 22). The logistic distribution is similar to the ND, but it has somewhat heavier tails. [For the logistic distribution, as $|\ell| \rightarrow \infty, f(\ell) \rightarrow e^{-|\ell|}$, whereas for the ND, as $|z| \rightarrow \infty$, $f(z) \rightarrow e^{-1 / 2 z^{2}}$.]

Although the chief reason for the popularity of the logistic transform is its ease of computation, another reason may be its slight advantage over the ND, which is due to the fact that empirical data often have heavier tailed distributions.

Alternatives to the ND like those discussed should be of particular interest when the tails of the distribution are in focus. One example is the use of response proportions for the scaling of perceived stimulus differences.

\section{APPLICATIONS TO DISTRIBUTIONS OF SUBJECTIVE QUANTITIES IN STIMULUS COMPARISON}

\section{Scaling Subjective Stimulus Differences}

As was first noted by Fechner (1860), systematic " timeorder errors" (TOEs) arise when two successive stimuli are compared. To study the mechanisms underlying TOEs, Hellström $(1977 \mathrm{a}, 1977 \mathrm{~b}, 1978,1979)$ did a series of experiments with paired successive stimuli. In the 1979 study, $1000-\mathrm{Hz}$ tones were compared for loudness. For each of 16 combinations of interstimulus interval (ISI) and tone duration, a different group of 12 subjects compared 16 tone pairs six times. Each pair had a different combination of sound-pressure levels of the two tones. The judgment alternatives were "first louder," "equal," and "second louder."

As will be described below, this three-category approach was used to obtain from the "equal" category a measuring stick for the subjective difference $d$ between the stimuli in a pair, namely, the half-width $T$ of the "equal" category. In a three-category comparison experiment with stimulus comparisons, $T$ determines the readiness to judge two stimuli with a small subjective difference as being equal. $T$ may vary from trial to trial as well as from subject to subject. The considerable variation between subjects in their tendency to pass "equal" or 
"doubtful" judgments, an obvious occurrence in Hellström's experiments, is a major reason why it has generally been thought best to avoid the three-category version of the method of constant stimulus differences (see, e.g., Woodworth \& Schlosberg, 1954, p. 217). However, this remark by Watson, Kellogg, Kawanishi, and Lukas (1973) may be applicable here as well as in the detection context: "Differences in the proportion of uncertain responses appear to reflect the same sort of sensitivity-independent effects as are found when the S's criterion is systematically varied in a yes-no experiment. Thus the more objective goal of pure sensitivity measurement may be achieved while allowing Ss to use the more subjective response set yes-no-maybe"' (p. 185).

In Hellström's (1977a, 1977b, 1978, 1979) measurement approach, $d$ is assumed to be ND over subjects and over trials, with mean $D$. Two response criteria are also assumed to exist on the $d$ continuum, with momentary positions ${ }^{1}$ $\tau_{1}>\tau_{2}$, so that the subject responds "first louder" when $d>\tau_{1}$, "equal" when $\tau_{2}<d<\tau_{1}$, and "second louder" when $d<\tau_{2} . \tau_{1}$ and $\tau_{2}$ are both assumed to be ND with variance $\sigma_{\tau}^{2}$ and (response-bias being ruled out as an important factor; see Hellström, 1977b, 1978) with expected values symmetric about zero: $E\left(\tau_{1}\right)=T, E\left(\tau_{2}\right)=-T$. Setting $c_{1}=d-\tau_{1}$ and $c_{2}=d-\tau_{2}$ yields $\sigma_{c_{1}}^{2}=\sigma_{d}^{2}+\sigma_{\tau_{1}}^{2}$ $-2 \operatorname{cov}\left(d, \tau_{1}\right)$ and $\sigma_{c_{2}}^{2}=\sigma_{d}^{2}+\sigma_{\tau_{2}}^{2}-2 \operatorname{cov}\left(d, \tau_{2}\right)$. On these assumptions, $c_{1}$ and $c_{2}$ are $\mathrm{ND}$, and if the two covariance terms are equal (they are both probably close to zero), we have $\sigma_{c_{1}}^{2}=\sigma_{c_{2}}^{2}=\sigma_{c}^{2}$.

The proportions $p_{1}$ and $p_{2}$ of "first louder" and "second louder" in the pooled data from each group and stimulus pair are transformed to the corresponding normal deviates $z_{1}$ and $z_{2}$ [i.e., $z_{1}=F^{-1}\left(p_{1}\right)$ and $z_{2}=-F^{-1}\left(p_{2}\right)$ ]. The following consistent estimates can then be made of $D$ and $\sigma_{c}$ (Hellström, 1977b; cf. Greenberg, 1965; Sjöberg, 1967):

$$
\begin{aligned}
& \left(D / \sigma_{c}\right)^{*}=\left(z_{1}+z_{2}\right) / 2 \\
& \left(\sigma_{c} / T\right)^{*}=2 /\left(z_{2}-z_{1}\right) \\
& (D / T)^{*}=\left(z_{2}+z_{1}\right) /\left(z_{2}-z_{1}\right) .
\end{aligned}
$$

Thurstone's method of pairwise comparisons, built on his law of comparative judgment (Thurstone, 1927a), involves the use of an equation similar to Equation 1, but it excludes "equal" judgments; therefore, $z_{1}$ and $z_{2}$ coincide, making $\left(D / \sigma_{c}\right)^{*}$ equal to the $z$ value that corresponds to the proportion of "greater." In the commonly used Case V of the law, $\sigma_{c}$ is assumed to be constant over pairs. $d$ is assumed to be ND over subjects; however, the method seems quite robust to violations of this assumption. Subkoviak (1974) found that " the amount of error in derived values increased as the degree of non-normality increased. However, the magnitude of error did not reach serious proportions except for distributions that were both extremely nonnormal and heterogeneous in shape" (p. 829).

The scaling method of Equations 1-3 may be regarded as a special case (with pairs of stimuli as objects to be categorized) of the law of categorical judgment (Torgerson, 1958, chap. 10), which in turn is a generalization (not assuming fixed category boundaries) of the method of successive intervals/categories (Guilford, 1954, chap. 10).

Similar scaling procedures for three-category comparisons were devised by Glenn and David (1960), Greenberg (1965), Olson and Ogilvie (1972), Saito (1988), and Sjöberg (1967). These authors scaled all the pairs simultaneously in accordance with certain assumptions, most importantly that $\sigma_{c}$ is constant over stimulus pairs. If this assumption is true, these procedures should be more satisfactory than the use of Equations 1-3. However, $\sigma_{c}$ must depend on the variabilities and intercorrelation of the compared subjective quantities, and these parameters are likely to differ between pairs of different composition.

For Hellström (1977a, 1977b, 1978, 1979), a reasonably constant-sized measuring stick for $D$ was an essential tool for his psychophysical modeling approach. He therefore preferred to scale each pair independently. Although Hellström's results indicated that $\left(D / \sigma_{c}\right)^{*}$ has less sampling variability than $(D / T)^{*}$, he used the latter for model fitting on the assumption that its unit $T$, the mean subjective half-width of the "equal" category, was approximately constant, or at least more so than $\sigma_{c}$, over stimulus magnitudes and temporal conditions used in the same session or for similar groups of subjects.

\section{Modeling Comparisons of Successive Stimuli}

To account for his successive-stimulus comparison results, Hellström (1977a, 1977b, 1978, 1979) devised a set of linear sensation weighting models. The basic model is as follows:

$$
d_{i k}=W_{1_{k}} \psi_{1}-W_{2_{k}} \psi_{2}+U_{k},
$$

where $\psi_{1}$ and $\psi_{2}$ are the sensation magnitudes of the stimuli in pair $i, W_{1_{k}}, W_{2_{k}}$, and $U_{k}$ their weighting coefficients and the intercept in condition $k . d_{i k}$ is the resulting momentary subjective stimulus difference for pair $i$ in condition $k$. Assuming (by Stevens's, 1957, power law) that $E \psi=\alpha \phi^{\beta}$, where $\phi$ is the physical magnitude of the stimulus and $E \psi$ the expected magnitude of the sensation it evokes, the following model was fitted by the iterative nonlinear function-fitting program STEPIT (Chandler, $1969)$ to the $(D / T)^{*}$ values:

$$
D_{i k} / T_{k}=B_{1_{k}} \phi_{1_{i}}^{\beta}-B_{2_{k}} \phi_{2_{i}}^{\beta}+C_{k},
$$

where $\phi_{1_{i}}$ and $\phi_{2_{i}}$ are the physical magnitudes of the stimuli in pair $i$, and $B_{1_{k}}, B_{2_{k}}$, and $C_{k}$ the weights and intercept for condition $k$. $D_{i k}$ is the mean subjective stimulus difference for pair $i$ in condition $k$, and $T_{k}$ the mean equalcategory half-width in condition $k$.

Hellström (1979) also computed $\left(\sigma_{c} / T\right)^{*}$ for each of the 16 tone pairs; this measure varied considerably between the 16 temporal conditions of stimulus presentation. ${ }^{2}$ Most of this variation could be explained within Hellström's theoretical framework as a dependence of $\sigma_{c}$ on the relation between the stimulus weights in the fitted model, and 
thus also supported the constancy assumption for $T$ over conditions (subject groups). In Hellström (1977a), with $T$ assumed to be constant over duration levels, results indicated the approximately linear psychophysical function for duration that was expected. Further support for the constancy of $T$ over stimulus levels is provided by the fact that the proportion of "equal" judgments tends to decline, and thus $\left(\sigma_{c} / T\right)^{*}$ tends to increase as predicted by Ekman's $(1956,1959)$ law, with increasing stimulus level within an experimental session; see Hellström (1977a, 1977b, 1978, 1989).

Although Equation 4 fit Hellström's (1979) $(D / T)^{*}$ values for loudness comparison very well $(3.50 \%$ of the total variance unexplained), the tone pairs with the largest physical stimulus differences caused a problem. For those pairs, the "minority" judgments (e.g., "second louder" with a stimulus difference favoring "first louder") were more numerous than expected. This decreased $z_{2}-z_{1}$, and consequently inflated $(D / T)^{*}$ and $\left(\sigma_{c} / T\right)^{*}$ to quite unrealistic values. A similar effect on loudness comparisons was demonstrated in Figure 4 of Hellström (1978); the data are replotted here as Figure 1. To display the effect more clearly, $z_{2}$ is plotted against $z_{1}$, resulting in a curvilinear plot. The reason for this effect was obscure, although Hellström (1978) suspected that "subjective difference distributions with extreme means ... are skewed with their longer tails pointing towards zero, resulting in reduced values of the extreme normal deviates"' (pp. 438-439). A similar effect can be found in Petrusic's (1981) duration comparison data, some of which are plotted in Figure 2. In Figures 1 and 2, in addition to the $z$ values, the corresponding $t$ values with $d f=2.5$ are plotted. As can be seen, this straightens out the plots quite efficiently. (The $d f$ value, selected after trying out several values, may still not be optimal.)

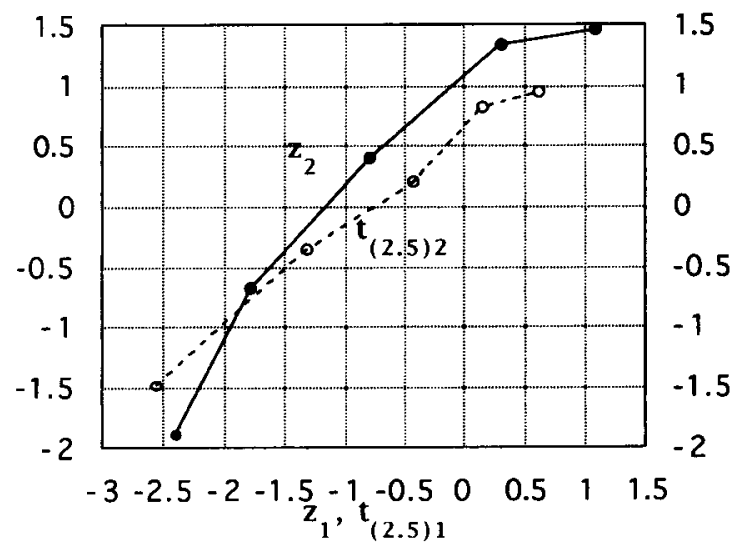

Figure 1. Loudness comparison data from Hellström (1978). Plots of normal deviates and values of $t(2.5)$ for proportions of "first longer" or "equal" $\left[z_{2}, t(2.5)_{2}\right]$ against corresponding values for proportions of "first longer" $\left[z_{1}, t(2.5)_{1}\right]$. Standard tone $70 \mathrm{~dB}$ SPL, comparison tones (data points from left to right) $66,68,70,72$, and $74 \mathrm{~dB}$ SPL.

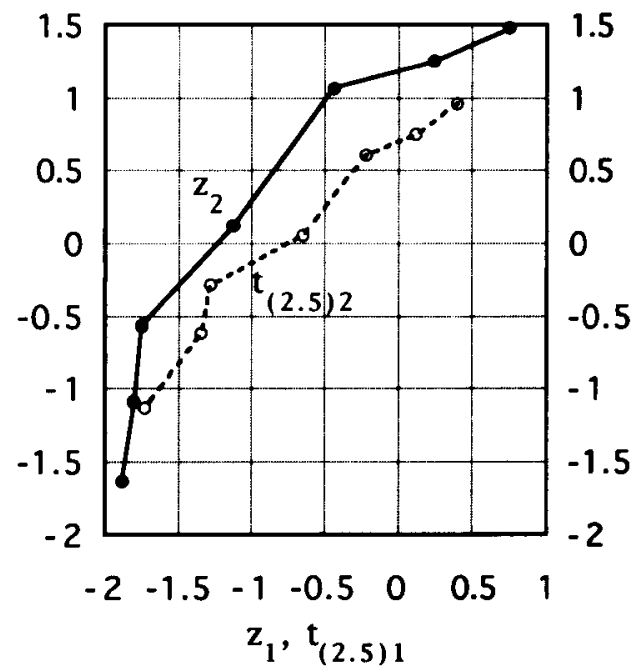

Figure 2. Duration comparison data from Petrusic (1981), Experiment 2: explicit counting, presentation order standard variable. Plots of normal deviates and values of $t(2.5)$ for proportions of "first longer" or "equal" $\left[z_{2}, t(2.5)_{2}\right]$ against corresponding values for proportions of "first longer" $\left[z_{1}, t(2.5)_{1}\right]$. Standard: 4,000 msec; comparison stimuli (data points from left to right): $3,400,3,600,3,800$, $4,000,4,200,4,400$, and $4,600 \mathrm{msec}$.

\section{Factors Behind the Nonnormality of Empirical $d$ Distributions}

In a comparison experiment like those producing the data plotted in Figures 1 and 2, assume that a linear model like Equation 4 holds, that the stimulus weights are constant, and that $\psi_{1}$ and $\psi_{2}$ are ND. $d$ should then also be ND. However, between subjects as well as between trials, the attention level and thereby also $\sigma_{c}$ may fluctuate; the stimulus weights $s_{1}$ and $s_{2}$ may also vary. This, together with variation of $T$ over subjects as well as trials, should yield a corresponding variation in the $S D$ of the momentary experienced stimulus difference $d$, and in the degree of precision in categorizing this difference. This is likely to cause an empirical $d$ distribution that can be modeled as a mixture of NDs with different $S D$ s and that is therefore heavy-tailed in comparison with the ND. In this type of situation, the $t$ distribution may be descriptively useful.

\section{IMPROVING MODEL FIT BY USING $t$ TO RESCALE SUBJECTIVE LOUDNESS DIFFERENCES}

As discussed above, the distribution of $d$ might be described better by $t$ with a small number of $d f$, rather than by the ND. This should then also be true for the distributions of $c_{1}=d_{1}-\tau_{1}$ and of $c_{2}=d_{2}-\tau_{2}$. Therefore, the use of $t$ in lieu of $z$ was expected to improve the scaling of $(D / T)^{*}$.

If a fitted model is correct, better scale values should improve the fit, but worse scale values should worsen it. If the model is wrong, its good fit has resulted from capitalizing on chance, and better scale values (actually, most 
Table 1

Total Percentage of Variance Unexplained by Equation 5 Fitted to Data From Hellström's (1979) Loudness Comparison Experiment, Using Values of $(D / T) *$ Computed From z, From $t$ With Various Degrees of Freedom (df), and From Logits

\begin{tabular}{cc}
$d f$ & $\begin{array}{c}\text { Unexplained } \\
\text { Variance, } \\
\%\end{array}$ \\
\hline 2.5 & 3.2242 \\
3.0 & 3.1231 \\
3.5 & 3.0850 \\
3.9 & 3.0768 \\
4.0 & 3.0767 \\
4.1 & 3.0771 \\
4.2 & 3.0777 \\
4.5 & 3.0824 \\
5.0 & 3.0953 \\
$\infty(=z)$ & 3.5013 \\
$\operatorname{logits}$ & 3.3584 \\
\hline
\end{tabular}

changes in the procedure) are likely to worsen the fit. Equation 5 fits Hellström's (1979) loudness comparison data closely and seems firmly anchored theoretically (Hellström, 1985, 1989). Therefore, it was expected that using the new values of $(D / T)^{*}$ would still improve the fit of this model.

\section{Method}

The $(D / T)^{*}$ values from Hellström (1979) were scaled, using $z$ as well as $t$ with various $d f$. Standard approximation formulas were used for the inverse normal and inverse $t$ distributions (Abramowitz \& Stegun, 1965, Equations 26.2.23 and 26.7.5); the $t$ values were rescaled to an $S D$ of 1 . STEPIT then fitted Equation 5 to the $(D / T)^{*}$ values.

\section{Results and Discussion}

Table 1 shows the percentage of total unexplained variance, $V \%_{\text {tot }}$, in fitting Equation 5 as a function of the $d f$ in the $t$ distribution used in modeling $(D / T)^{*}$. Regarding the $d f$ as an additional estimated parameter, the improvement in the fit caused by its introduction is highly significant $[F(1,214)=19.91, p=.000013]$. This improvement, a $12.1 \%$ reduction in $V \%_{\text {tot }}$, is yielded by $d f=$ 4.0 , which seems to be close to the optimum. (Thus, the optimal $d f$ value for these data is less extreme than the 2.5 required to linearize the plots in Figures 1 and 2.) Given the reasoning above, this result can be counted as support for Equation 5 as well as for using $t(4)$ in scaling the data to be fitted. Likewise, the use of logits yielded a slight improvement in the fit. Therefore, in the following, logits and $t$ with $d f=4$, computed and rescaled as above, and denoted by $t(4)$, will be evaluated as alternatives to the ND.

\section{USING THE $t$ DISTRIBUTION TO LINEARIZE THE RELATION BETWEEN DEVIATES}

Let us take a closer look at the inflation effect that occurs when the ND is used. In a pilot experiment (Experiment 1, Group 1) for his 1979 study, Hellström (1977a) had 20 subjects compare 25 tone pairs (tone duration $1 \mathrm{sec}$, ISI $1.5 \mathrm{sec}$ ) five times each. Assuming $d$ to be ND and using Equations 2 and $3,(D / T)^{*},\left(\sigma_{c} / T\right)^{*}$, and $\left(D / \sigma_{c}\right)^{*}$ were computed from the $z$ values for each pair. The results are given in Table 2 , together with the cor-

Table 2

Data From Hellström (1977a), Experiment 1 (Group 1): Judgment Distributions and Scale Values, Computed From z, Logits $(\ell)$ and $t(4)$

\begin{tabular}{|c|c|c|c|c|c|c|c|c|c|c|c|c|c|}
\hline \multirow[b]{3}{*}{$M$ SPL* } & \multirow{3}{*}{$\begin{array}{l}\text { SPL Diff. } \\
\text { Between 1st } \\
\text { and 2nd Tone* }\end{array}$} & \multicolumn{3}{|c|}{ Judgment } & \multirow{2}{*}{\multicolumn{3}{|c|}{$z$}} & \multirow{2}{*}{\multicolumn{3}{|c|}{ e }} & \multirow{2}{*}{\multicolumn{3}{|c|}{$t(4)$}} \\
\hline & & $1 \mathrm{st}$ & & $" 2$ & & & & & & & & & \\
\hline & & Louder"' & "Equal" & Louder", & $(D / T)^{*}$ & $\left(\sigma_{c} / T\right)^{*}$ & $\left(D / \sigma_{c}\right)^{*}$ & $(D / T)^{*}$ & $\left(\sigma_{c} / T\right)^{*}$ & $\left(D / \sigma_{c}\right)^{*}$ & $(D / T)^{*}$ & $\left(\sigma_{c} / T\right)^{*}$ & $\left(D / \sigma_{c}\right)^{*}$ \\
\hline 71.6 & $\begin{array}{r}-3.2 \\
-1.6 \\
0.0 \\
+1.6 \\
+3.2\end{array}$ & $\begin{array}{r}0 \\
1 \\
7 \\
52 \\
92\end{array}$ & $\begin{array}{r}6 \\
40 \\
80 \\
45 \\
7\end{array}$ & $\begin{array}{r}94 \\
59 \\
13 \\
3 \\
1\end{array}$ & $\begin{array}{r}-1.22 \\
-0.13 \\
1.06 \\
4.05\end{array}$ & $\begin{array}{l}0.95 \\
0.77 \\
1.09 \\
2.17\end{array}$ & $\begin{array}{r}-1.28 \\
-0.17 \\
0.97 \\
1.87\end{array}$ & $\begin{array}{r}-1.17 \\
-0.15 \\
1.05 \\
3.27\end{array}$ & $\begin{array}{l}0.86 \\
0.80 \\
1.07 \\
1.69\end{array}$ & $\begin{array}{r}-1.37 \\
-0.19 \\
0.98 \\
1.94\end{array}$ & $\begin{array}{r}-1.14 \\
-0.17 \\
1.04 \\
2.71\end{array}$ & $\begin{array}{l}0.81 \\
0.90 \\
1.11 \\
1.40\end{array}$ & $\begin{array}{r}-1.41 \\
-0.19 \\
0.94 \\
1.93\end{array}$ \\
\hline 70.8 & $\begin{array}{r}-3.2 \\
-1.6 \\
0.0 \\
+1.6 \\
+3.2\end{array}$ & $\begin{array}{r}0 \\
1 \\
10 \\
52 \\
91\end{array}$ & $\begin{array}{r}9 \\
41 \\
74 \\
46 \\
7\end{array}$ & $\begin{array}{r}91 \\
58 \\
16 \\
2 \\
2\end{array}$ & $\begin{array}{r}-1.19 \\
-0.13 \\
1.05 \\
4.76\end{array}$ & $\begin{array}{l}0.94 \\
0.88 \\
1.00 \\
2.80\end{array}$ & $\begin{array}{r}-1.26 \\
-0.14 \\
1.05 \\
1.70\end{array}$ & $\begin{array}{r}-1.15 \\
-0.14 \\
1.04 \\
3.93\end{array}$ & $\begin{array}{l}0.85 \\
0.94 \\
0.95 \\
2.30\end{array}$ & $\begin{array}{r}-1.36 \\
-0.15 \\
1.09 \\
1.71\end{array}$ & $\begin{array}{r}-1.12 \\
-0.15 \\
1.04 \\
3.36\end{array}$ & $\begin{array}{l}0.80 \\
1.06 \\
0.96 \\
2.06\end{array}$ & $\begin{array}{r}-1.40 \\
-0.14 \\
1.08 \\
1.63\end{array}$ \\
\hline 70.0 & $\begin{array}{r}-3.2 \\
-1.6 \\
0.0 \\
+1.6 \\
+3.2\end{array}$ & $\begin{array}{r}0 \\
0 \\
16 \\
66 \\
94\end{array}$ & $\begin{array}{r}14 \\
53 \\
72 \\
33 \\
4\end{array}$ & $\begin{array}{r}86 \\
47 \\
12 \\
1 \\
2\end{array}$ & $\begin{array}{l}0.08 \\
1.43 \\
7.23\end{array}$ & $\begin{array}{l}0.92 \\
1.04 \\
4.01\end{array}$ & $\begin{array}{l}0.09 \\
1.37 \\
1.80\end{array}$ & $\begin{array}{l}0.09 \\
1.34 \\
5.83\end{array}$ & $\begin{array}{l}0.99 \\
0.92 \\
3.18\end{array}$ & $\begin{array}{l}0.09 \\
1.45 \\
1.83\end{array}$ & $\begin{array}{l}0.10 \\
1.27 \\
4.84\end{array}$ & $\begin{array}{l}1.13 \\
0.86 \\
2.75\end{array}$ & $\begin{array}{l}0.09 \\
1.48 \\
1.76\end{array}$ \\
\hline 69.2 & $\begin{array}{r}-3.2 \\
-1.6 \\
0.0 \\
+1.6 \\
+3.2\end{array}$ & $\begin{array}{r}1 \\
2 \\
17 \\
68 \\
96\end{array}$ & $\begin{array}{r}13 \\
55 \\
73 \\
30 \\
4\end{array}$ & $\begin{array}{r}86 \\
43 \\
10 \\
2 \\
0\end{array}$ & $\begin{array}{r}-2.73 \\
-0.84 \\
0.15 \\
1.59\end{array}$ & $\begin{array}{l}1.60 \\
0.90 \\
0.89 \\
1.26\end{array}$ & $\begin{array}{r}-1.70 \\
-0.94 \\
0.16 \\
1.26\end{array}$ & $\begin{array}{r}-2.31 \\
-0.86 \\
0.16 \\
1.48\end{array}$ & $\begin{array}{l}1.30 \\
0.87 \\
0.96 \\
1.16\end{array}$ & $\begin{array}{r}-1.77 \\
-1.00 \\
0.17 \\
1.28\end{array}$ & $\begin{array}{r}-2.00 \\
-0.88 \\
0.17 \\
1.40\end{array}$ & $\begin{array}{l}1.13 \\
0.89 \\
1.08 \\
1.13\end{array}$ & $\begin{array}{r}-1.77 \\
-0.99 \\
0.16 \\
1.24\end{array}$ \\
\hline 68.4 & $\begin{array}{r}-3.2 \\
-1.6 \\
0.0 \\
+1.6 \\
+3.2\end{array}$ & $\begin{array}{r}2 \\
4 \\
17 \\
71 \\
98\end{array}$ & $\begin{array}{r}15 \\
64 \\
74 \\
26 \\
2\end{array}$ & $\begin{array}{r}83 \\
32 \\
9 \\
3 \\
0\end{array}$ & $\begin{array}{r}-2.73 \\
-0.58 \\
0.17 \\
1.83\end{array}$ & $\begin{array}{l}1.82 \\
0.90 \\
0.87 \\
1.51\end{array}$ & $\begin{array}{r}-1.50 \\
-0.64 \\
0.19 \\
1.22\end{array}$ & $\begin{array}{r}-2.38 \\
-0.62 \\
0.19 \\
1.69\end{array}$ & $\begin{array}{l}1.57 \\
0.92 \\
0.93 \\
1.41\end{array}$ & $\begin{array}{r}-1.51 \\
-0.67 \\
0.20 \\
1.21\end{array}$ & $\begin{array}{r}-2.13 \\
-0.64 \\
0.20 \\
1.60\end{array}$ & $\begin{array}{l}1.48 \\
1.00 \\
1.05 \\
1.41\end{array}$ & $\begin{array}{r}-1.44 \\
-0.65 \\
0.19 \\
1.13\end{array}$ \\
\hline
\end{tabular}

*In decibels. 
responding values computed from $t(4)$ and logits $(\ell)$. (For six pairs, there were no judgments in one of the categories; those pairs are omitted.) Using $z$ yields $(D / T)^{*}$ and $\left(\sigma_{c} / T\right)^{*}$ values that are heavily inflated [unlike $\left(D / \sigma_{c}\right)^{*}$ ] for the larger stimulus differences. This effect diminishes when $t(4)$ is used instead. To a smaller extent, using logits yields the same result. Figures 3-5 illustrate the origin of the inflation effect. The plot of $z_{2}$ against $z_{1}$ (Figure 3 ) is curved because these values approach each other, the more extreme value lagging behind, when their absolute

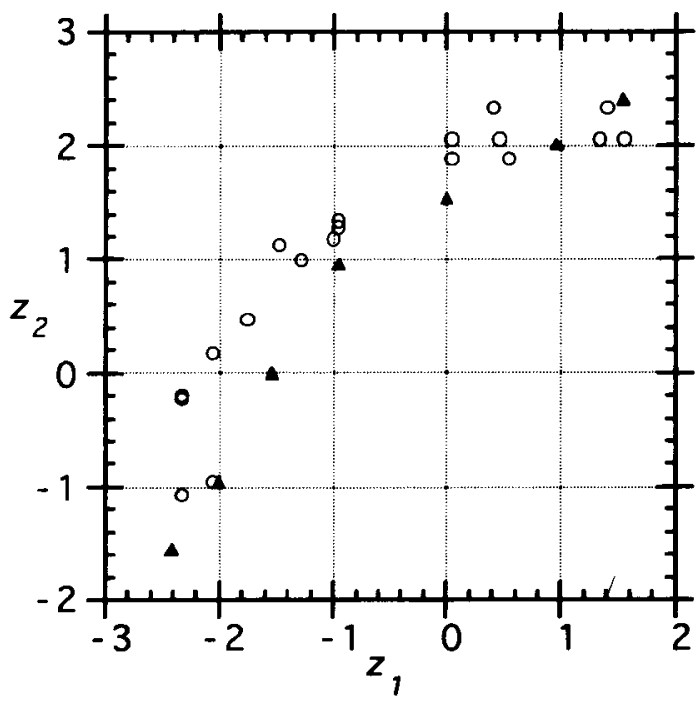

Figure 3. Plots of normal deviates ( $z$ values). Open circles: plot of $z_{2}$ against $z_{1}$ for data from Hellström (1977a), Experiment 1, Group 1 (cf. Table 2). Solid triangles: plot of $z_{2}$ against $z_{1}$ for simulation results with $\sigma_{d}=1, \sigma_{T}=0.0, \sigma_{\sigma}=0.8$ (Table $3 C$ ).

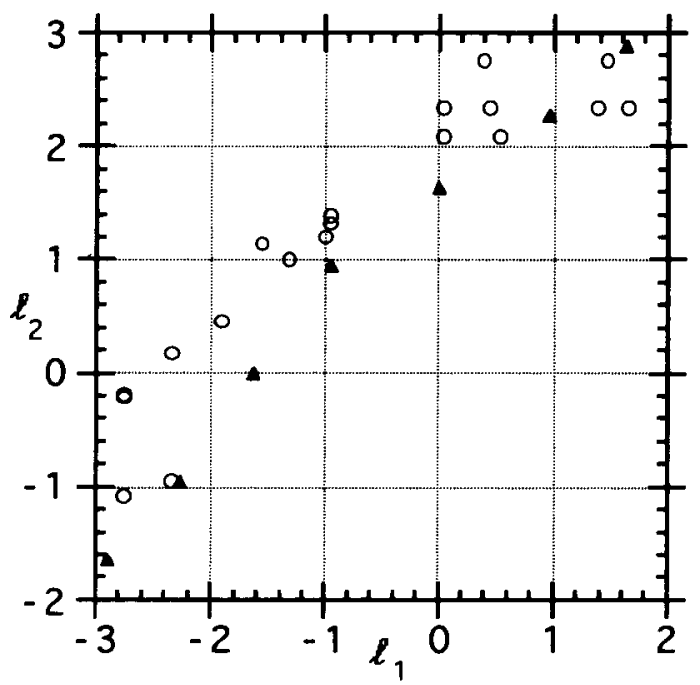

Figure 4. Plots of logits ( $\ell$ values; rescaled to $\sigma_{\ell}=1$ ). Open circles: plot of $\ell_{2}$ against $\ell_{1}$ for data from Hellström (1977a), Experiment 1, Group 1 (cf. Table 2). Solid triangles: plot of $\ell_{2}$ against $\ell_{1}$ for simulation results with $\sigma_{d}=1, \sigma_{T}=0.0, \sigma_{\sigma}=0.8$ (Table 3C).

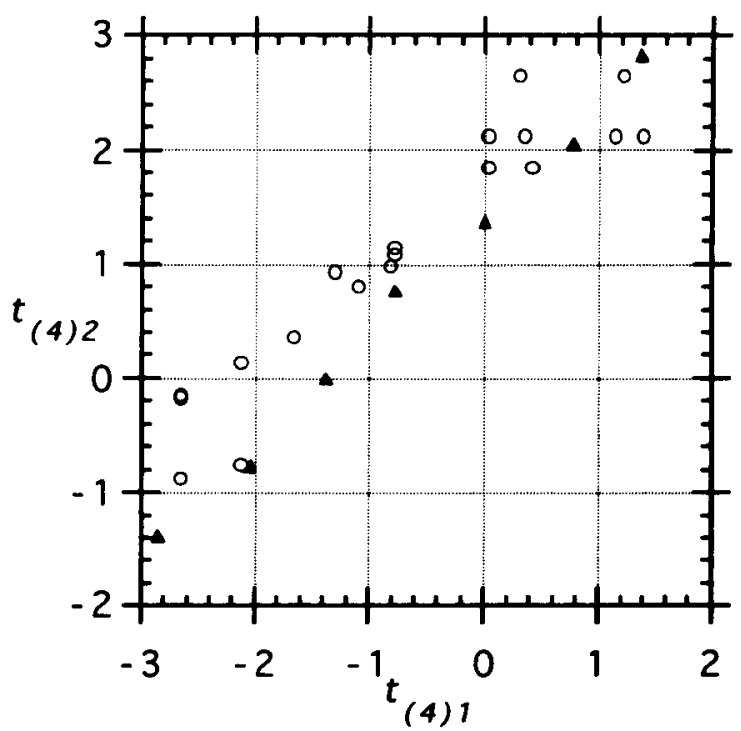

Figure 5. Plots of $t(4)$ deviates (rescaled to $\sigma_{t}=1$ ). Open circles: plot of $t(4)_{2}$ against $t(4)_{1}$ for data from Hellström (1977a), Experiment 1 , Group 1 (cf. Table 2). Solid triangles: plot of $t(4)_{2}$ against $t(4)$, for simulation results with $\sigma_{d}=1, \sigma_{T}=0.0, \sigma_{\sigma}=0.8$ (Table 3C).

values increase (cf. Figures 1-2). This means that the absolute value of $\left(D / \sigma_{c}\right)^{*}=\left(z_{1}+z_{2}\right) / 2$ will also be lagging behind its "true" value.) In contrast, the plot of $t(4)_{2}$ against $t(4)_{1}$ (Figure 5) is more linear and parallel to the diagonal, indicating that their difference is more constant over stimulus pairs. The characteristics of the logit plot (Figure 4) fall in between those of the $z$ and $t(4)$ plots. (The triangles are values from the Monte Carlo study, discussed below.)

Bias due to selection. Some of the extreme pairs diverge somewhat from the trend of the others, even in the $t(4)$ plot (Figure 4); their largest (absolute) deviate value is too small. $\sigma_{d}$ may be somewhat greater for these pairs than for the others (e.g., because of lower intercorrelations between the compared $\psi$ values); however, this should change both deviates by similar factors. A more likely reason for the diversions is random scattering and subsequent biased selection; for a pair to get a scale value at all, it must have at least one judgment in each category. For a pair with a large difference between the stimulus magnitudes, this requires that the minority judgments are more numerous than expected. Consequently, the absolute values of the extreme deviates (e.g., $z$ ) have to be lower than expected, and $(D / T)^{*}$ and $\left(\sigma_{c} / T\right)^{*}$ higher than expected. For example, assume that a stimulus pair has the "true" values $(D / T)=+2$ and $\left(\sigma_{c} / T\right)=1$. Applying Equations 2 and 3 backwards yields $z_{1}=+1$ and $z_{2}=$ +3 , which for the ND case corresponds to a probability of .841 for "first louder," .157 for "equal," and .00135 for "second louder." In 100 judgments, the probability of at least one "second louder," and thus of a resulting scale value, then becomes .126. In $94 \%$ of these cases, there will be exactly one "second louder," making $z_{2}=$ 
2.33. For such a case, if one assumes 84 judgments of "first louder" and 15 of "equal," $z_{1}$ becomes .994, making $(D / T)^{*}$ increase to 2.49 and $\left(\sigma_{c} / T\right)^{*}$ to 1.50 , whereas $\left(D / \sigma_{c}\right)^{*}$ decreases to 1.66 . Bringing $(D / T)^{*}$ down to +2 requires that $z_{1}$ decrease to 0.776 and thus the number of "first louder" to 78 , leaving 21 judgments of "equal."

The 100-judgment situation was computer simulated in 100,000 replications, 12,124 of which yielded at least one judgment of "second louder" and thus a scale value. For these replications, the mean number of judgments was 81.93 for "first louder," 16.96 for "equal," and 1.11 for "second louder," and the mean values of $(D / T)^{*}$, $\left(\sigma_{c} / T\right)^{*}$, and $\left(D / \sigma_{c}\right)^{*}$ were $2.38,1.47$, and 1.61 .

The direction of the selection effect on $(D / T)^{*}$ may seem paradoxical, an additional minority judgment changing the scale value in favor of the majority judgments. However, with the proportion of "second louder" judgments denoted as $p_{2}$, the effect can be predicted thus (here we assume that the sample of judgments is infinitely large; $H$ and $h$ denote the distribution and density functions of the underlying distribution):

$$
\begin{aligned}
& \mathrm{d}(D / T)^{*} / \mathrm{d} p_{2}=\partial(D / T)^{*} / \partial z_{2} \cdot \mathrm{d} z_{2} / \mathrm{d} p_{2} \\
& =2 z_{1} /\left[\left(z_{2}-z_{1}\right)^{2} \cdot h\left(z_{2}\right)\right],
\end{aligned}
$$

where $z_{2}=-H^{-1}\left(p_{2}\right)$. The corresponding results for $\left(\sigma_{c} / T\right)^{*}$ and $\left(D / \sigma_{c}\right)^{*}$ are

$$
\mathrm{d}\left(\sigma_{\mathrm{c}} / T\right)^{*} / \mathrm{d} p_{2}=2\left[\left(z_{2}-z_{1}\right)^{2} \cdot h\left(z_{2}\right)\right]
$$

and

$$
\mathrm{d}\left(D / \sigma_{c}\right) * / \mathrm{d} p_{2}=-1 /\left[2 h\left(z_{2}\right)\right]
$$

Thus, with a small proportion of minority judgments, fluctuations in this proportion have a disproportionate and biasing impact on the scale values, particularly for $(D / T)^{*}$ and $\left(\sigma_{c} / T\right)^{*}$. This means that pairs with too few minority judgments should be excluded from the computationsat the very least, the pairs for which the number of minority judgments is 1 .

\section{A MONTE CARLO STUDY}

The preceding results support the notion that the inflated $(D / T)^{*}$ and $\left(\sigma_{c} / T\right)^{*}$ values were caused by two factors: (1) between-subject variability of $\sigma_{c}$, yielding a pooled inter- and intraindividual distribution of $d$ that is heavytailed in comparison with the ND and that can be approximated by $t$ with a small number of $d f$; and (2) random fluctuation of minority judgments and ensuing biased selection of pairs with spuriously high values of $(D / T)^{*}$ and $\left(\sigma_{c} / T\right)^{*}$.

To explore this matter further, and to identify factors likely to shape the $d$ distribution, while eliminating the problems resulting from too few judgments, a comparison experiment was Monte Carlo simulated.

For pooled data from several subjects, we may consider $D, T$, and $\sigma_{c}$ as averages of the individual mean values $D_{i}, T_{i}$, and $\sigma_{c_{i}}$. For lack of more plausible hypoth- eses, $D_{i}$ and $T_{i}$ may be assumed to be ND over subjects. A nonzero $\sigma_{T}$ also implies that the scale unit of $D / T$ varies. As for $\sigma_{c}$, it is composed of the total variabilities of $d_{i}$ and $T_{i}$. Thus, interindividual variation in $\sigma_{d}$ as well as in $T$ makes the total distribution used to scale $D / T$ and $D / \sigma_{c}$ a mixture of distributions with different variabilities. The effects of these factors were studied by simulation.

\section{Method}

In 10,000 replications, an experiment similar to Hellström's (1977a) Experiment 1, Group 1, was simulated. In the simulation, 20 subjects compared the stimuli in each of four pairs five times, using the categories "first greater," "equal," and "second greater." The design was as follows.

For each stimulus pair, the mean or "true" value of the subjective stimulus difference was set to $D(=0,+1,+2$, or +3$)$.

The momentary (trial-specific) subjective stimulus difference was made a stochastic variable represented by the pseudorandom number $d$.

The response category limits for $d$ were $\tau_{1}=T_{i}$ and $\tau_{2}=-T_{i}$, where $T_{i}=$ the subject's individual half-width of the subjective "equal" category.

$T_{i}$ was fixed for each subject, and ND over subjects with mean = 1 and $S D=\sigma_{T}$. $\sigma_{T}$ was set to 0.0 or 0.8 (but with only positive values accepted). ${ }^{3}$

The relation of $d$ to $\tau_{1}$ and $\tau_{2}$ determined the response assumed above-that is, $d>\tau_{1}$ yielded "first louder," $\tau_{2}<d<\tau_{1}$ "equal," and $d<\tau_{2}$ "second louder."

For each subject, $d$ was ND over trials, with mean $=D$ and an individual $S D$ value $=\sigma_{d_{i}}$.

$\sigma_{d_{i}}$ was ND over subjects (but with only positive values accepted), with mean $=1$ and $S D=\sigma_{\sigma} . \sigma_{\sigma}$ was set to 0.0 or 0.8 .

$T_{i}$ and $\sigma_{d_{i}}$ were uncorrelated over subjects.

The 10,000 distributions of the $20 \cdot 5$ judgments were summed, making a total of $1,000,000$ judgments for each pair. $(D / T)^{*}$, $\left(\sigma_{c} / T\right)^{*}$, and $\left(D / \sigma_{c}\right)^{*}$ were calculated with Equations $1-3$, using $z$, logits $(\ell)$, and $t(4)$. ( $d f=4$ was selected because, as discussed above, this was the value found optimal for fitting Equation 5 to the data from Hellström, 1979, for which Experiment 1 of Hellström, 1977a, was a pilot experiment.)

\section{Results and Discussion}

The results for all combinations of the simulated values 0.0 and 0.8 of $\sigma_{T}$ and $\sigma_{\sigma}$ are shown in Tables 3A-3D. For each row of computed values, the slope of the linear regression on $D$ and the complement of the corresponding coefficient of determination $\left(1-R^{2}\right)$ are given. In addition, the quadratic and cubic trends are described, using standard orthogonal polynomials.

With $\sigma_{T}=\sigma_{\sigma}=0$ (Table 3A), and using $z,(D / T)^{*}$, $\left(D \sigma_{c}\right)^{*}$, and $\left(\sigma_{c} / T\right)^{*}$ accurately recover the true values of $D(0,1,2$, or 3$)$ and $\sigma_{d}(1)$. Also, using $z,(D / T)^{*}$ is quite resistant against interindividual variability of $T_{i}\left(\sigma_{T}=0.8\right.$, Table 3B), but not of $\sigma_{d_{i}}\left(\sigma_{\sigma}=0.8\right.$, Table 3C). Generally, $\left(D / \sigma_{c}\right)^{*}$ is more linear against $D$ than is $(D / T)^{*}$, and shows no inflation effect (quadratic trends close to zero), although its scale unit $\sigma_{c}$ increases with nonzero $\sigma_{\sigma}$ and $\sigma_{T}$. As expected, a nonzero $\sigma_{\sigma}$ causes the minority judgments to increase, making the two normal deviates $z_{1}$ and $z_{2}$ approach each other for the larger $D$ values, which in turn causes the inflation effect for $(D / T)^{*}$ and $\left(\sigma_{c} / T\right)^{*}$ 
Table 3A

Simulation Results: $\sigma_{T}=0.0, \sigma_{\sigma}=0.0$; Judgment Distributions;

Computed Measures; Trends

\begin{tabular}{|c|c|c|c|c|c|c|c|c|}
\hline & $D=0.0$ & $D=1.0$ & $D=2.0$ & $D=3.0$ & $1-R^{2}$ & Slope & Quadratic & Cubic \\
\hline "First" & 158482 & 500376 & 841046 & 977245 & & & & \\
\hline "Equal" & 682731 & 476749 & 157630 & 22735 & & & & \\
\hline "Second" & 158787 & 22875 & 1324 & 20 & & & & \\
\hline
\end{tabular}

Estimates based on normal distribution of subjective difference $d$ :

$\begin{array}{lrllllllr}z_{1} & -1.001 & 0.001 & 0.999 & 2.000 & 5.40 \mathrm{E}-7 & 1.000 & -.001 & .007 \\ z_{2} & 0.999 & 1.998 & 3.006 & 4.107 & 5.53 \mathrm{E}-4 & 1.033 & .102 & .084 \\ (D / T)^{*} & -0.001 & 1.001 & 1.995 & 2.899 & 5.82 \mathrm{E}-4 & .969 & -.098 & -.082 \\ \left(\sigma_{c} / T\right)^{*} & 1.000 & 1.001 & 0.996 & 0.949 & 3.39 \mathrm{E}-1 & -.016 & -.048 & -.036 \\ \left(D / \sigma_{c}\right)^{*} & -0.001 & 1.000 & 2.002 & 3.054 & 1.49 \mathrm{E}-4 & 1.017 & .051 & .049\end{array}$

Estimates based on logistic distribution of subjective difference $d$ :

$\begin{array}{lrllllrrr}\ell_{1} & -0.920 & 0.001 & 0.919 & 2.073 & 3.34 \mathrm{E}-3 & 0.990 & .233 & .239 \\ \ell_{2} & 0.919 & 2.070 & 3.653 & 5.964 & 2.38 \mathrm{E}-1 & 1.672 & 1.160 & .296 \\ (D / T)^{*} & -0.001 & 1.001 & 1.672 & 2.065 & 3.79 \mathrm{E}-2 & .687 & -.609 & .053 \\ \left(\sigma_{c} / T\right)^{*} & 1.087 & 0.967 & 0.731 & 0.514 & 1.68 \mathrm{E}-2 & -.196 & -.097 & .135 \\ \left(D / \sigma_{c}\right)^{*} & -0.001 & 1.035 & 2.286 & 4.019 & 1.39 \mathrm{E}-2 & 1.331 & .697 & .267\end{array}$

Estimates based on $t(4)$ distribution of subjective difference $d$ :

\begin{tabular}{lrlrrrrrr}
$t_{1}$ & -0.808 & 0.001 & 0.806 & 2.029 & $1.18 \mathrm{E}-2$ & .932 & .414 & .422 \\
$t_{2}$ & 0.807 & 2.025 & 4.675 & 13.020 & $1.50 \mathrm{E}-1$ & 3.929 & 7.127 & 4.263 \\
$(D / T)^{*}$ & -0.001 & 1.001 & 1.417 & 1.369 & $2.12 \mathrm{E}-1$ & .453 & -1.050 & .122 \\
$\left(\sigma_{c} / T\right)^{*}$ & 1.239 & 0.988 & 0.517 & 0.182 & $1.21 \mathrm{E}-2$ & -.364 & -.084 & .356 \\
$\left(D / \sigma_{c}\right)^{*}$ & -0.001 & 1.013 & 2.740 & 7.524 & $1.15 \mathrm{E}-1$ & 2.430 & 3.770 & 2.344 \\
\hline
\end{tabular}

Table 3B

Simulation Results: $\sigma_{T}=0.8, \sigma_{\sigma}=0.0 ;$ Judgment Distributions;

Computed Measures; Trends

\begin{tabular}{lrrrrrrr}
\hline & $D=0.0$ & $D=1.0$ & $D=2.0$ & $D=3.0$ & $1-R^{2}$ & Slope Quadratic & Cubic \\
\hline "First" & 167477 & 452296 & 758605 & 934193 & & & \\
"Equal" & 665228 & 514464 & 238229 & 65681 & & & \\
"Second" & 167295 & 33240 & 3166 & 126 & & &
\end{tabular}

Estimates based on normal distribution of subjective difference $d$ :

$\begin{array}{lrrrrrrrr}z_{1} & -0.964 & -0.120 & 0.702 & 1.508 & 1.07 \mathrm{E}-4 & -.824 & .038 & .006 \\ z_{2} & 0.965 & 1.836 & 2.730 & 3.660 & 2.18 \mathrm{E}-4 & .898 & .059 & .013 \\ (D / T)^{*} & 0.000 & 0.878 & 1.692 & 2.401 & 2.24 \mathrm{E}-3 & .802 & -.169 & -.041 \\ \left(\sigma_{c} / T\right)^{*} & 1.037 & 1.023 & 0.986 & 0.929 & 6.63 \mathrm{E}-2 & -.036 & -.043 & .003 \\ \left(D / \sigma_{c}\right)^{*} & 0.000 & 0.858 & 1.716 & 2.584 & 8.09 \mathrm{E}-6 & .861 & .010 & .010\end{array}$

Estimates based on logistic distribution of subjective difference $d$ :

$\begin{array}{lrrrrrrrr}\ell_{1} & -0.884 & -0.106 & 0.631 & 1.463 & 5.46 \mathrm{E}-4 & .778 & .054 & .136 \\ \ell_{2} & 0.885 & 1.858 & 3.171 & 4.950 & 1.76 \mathrm{E}-2 & 1.351 & .806 & .126 \\ (D / T)^{*} & 0.000 & 0.893 & 1.497 & 1.839 & 3.90 \mathrm{E}-2 & .612 & -.551 & .027 \\ \left(\sigma_{c} / T\right)^{*} & 1.131 & 1.019 & 0.787 & 0.573 & 1.92 \mathrm{E}-2 & -.191 & -.102 & .138 \\ \left(D / \sigma_{c}\right)^{*} & 0.000 & 0.876 & 1.901 & 3.207 & 8.24 \mathrm{E}-3 & 1.065 & .430 & .132\end{array}$

Estimates based on $t(4)$ distribution of subjective difference $d$ :

\begin{tabular}{lrrrrrrrr}
$t_{1}$ & -0.774 & -0.090 & 0.546 & 1.337 & $2.02 \mathrm{E}-3$ & .697 & .107 & .203 \\
$t_{2}$ & 0.775 & 1.771 & 3.695 & 8.448 & $1.07 \mathrm{E}-1$ & 2.494 & 3.757 & 1.901 \\
$(D / T)^{*}$ & 0.000 & 0.903 & 1.347 & 1.376 & $1.55 \mathrm{E}-1$ & .457 & -.874 & .044 \\
$\left(\sigma_{c} / T\right)^{*}$ & 1.291 & 1.075 & 0.635 & 0.281 & $1.56 \mathrm{E}-2$ & -.347 & -.138 & .310 \\
$\left(D / \sigma_{c}\right)^{*}$ & 0.000 & 0.840 & 2.121 & 4.893 & $7.20 \mathrm{E}-2$ & 1.596 & 1.932 & 1.050 \\
\hline
\end{tabular}


Table 3C

Simulation Results: $\sigma_{T}=0.0, \sigma_{\sigma}=0.8 ;$ Judgment Distributions;

Computed Measures; Trends

\begin{tabular}{lrrrrrrr} 
& $D=0.0$ & $D=1.0$ & $D=2.0$ & $D=3.0$ & $1-R^{2}$ & Slope Quadratic & Cubic \\
\hline "First" & 169454 & 500930 & 830986 & 938121 & & & \\
"Equal" & 661709 & 437403 & 147102 & 53890 & & & \\
"Second" & 168837 & 61667 & 21912 & 7989 & &
\end{tabular}

Estimates based on normal distribution of subjective difference $d$ :

$\begin{array}{lrllllllr}z_{1} & -0.956 & 0.002 & 0.958 & 1.540 & 1.17 \mathrm{E}-2 & .844 & -.376 & -.372 \\ z_{2} & 0.959 & 1.541 & 2.016 & 2.410 & 7.55 \mathrm{E}-3 & .483 & -.188 & .026 \\ (D / T)^{*} & 0.001 & 1.003 & 2.811 & 4.538 & 1.42 \mathrm{E}-2 & 1.542 & .725 & -.887 \\ \left(\sigma_{c} / T\right)^{*} & 1.044 & 1.300 & 1.890 & 2.298 & 1.98 \mathrm{E}-2 & .435 & .152 & -.516 \\ \left(D / \sigma_{c}\right)^{*} & 0.001 & 0.772 & 1.487 & 1.975 & 9.66 \mathrm{E}-3 & .664 & -.283 & -.171\end{array}$

Estimates based on logistic distribution of subjective difference $d$ :

$\begin{array}{lrllllllr}\ell_{1} & -0.876 & 0.002 & 0.878 & 1.499 & 6.12 \mathrm{E}-3 & .800 & -.257 & -.253 \\ \ell_{2} & 0.879 & 1.501 & 2.094 & 2.658 & 4.78 \mathrm{E}-4 & .593 & -.058 & .000 \\ (D / T)^{*} & 0.001 & 1.003 & 2.444 & 3.586 & 4.30 \mathrm{E}-3 & 1.220 & .140 & -.738 \\ \left(\sigma_{c} / T\right)^{*} & 1.140 & 1.334 & 1.644 & 1.725 & 4.11 \mathrm{E}-2 & .206 & -.113 & -.345 \\ \left(D / \sigma_{c}\right)^{*} & 0.001 & 0.751 & 1.486 & 2.079 & 2.86 \mathrm{E}-3 & .697 & -.157 & -.127\end{array}$

Estimates based on $t(4)$ distribution of subjective difference $d$ :

\begin{tabular}{lrllllllr}
$t_{1}$ & -0.767 & 0.002 & 0.769 & 1.375 & $3.05 \mathrm{E}-3$ & .719 & -.163 & -.159 \\
$t_{2}$ & 0.769 & 1.377 & 2.055 & 2.834 & $3.11 \mathrm{E}-3$ & .687 & .171 & .031 \\
$(D / T)^{*}$ & 0.001 & 1.003 & 2.195 & 2.886 & $9.81 \mathrm{E}-3$ & .985 & -.311 & -.691 \\
$\left(\sigma_{c} / T\right)^{*}$ & 1.302 & 1.454 & 1.555 & 1.371 & $8.67 \mathrm{E}-1$ & .031 & -.336 & -.234 \\
$\left(D / \sigma_{c}\right)^{*}$ & 0.001 & 0.690 & 1.412 & 2.104 & $8.12 \mathrm{E}-5$ & .703 & .003 & -.063 \\
\hline
\end{tabular}

Table 3D

Simulation Results: $\sigma T=0.8, \sigma_{\sigma}=0.8$; Judgment Distributions;

Computed Measures; Trends

\begin{tabular}{|c|c|c|c|c|c|c|c|c|}
\hline & $D=0.0$ & $D=1.0$ & $D=2.0$ & $D=3.0$ & $1-R^{2}$ & Slope & Quadratic & Cubic \\
\hline "First" & 158750 & 546751 & 770200 & 889994 & & & & \\
\hline "Equal", & 682601 & $\begin{array}{r}421853 \\
31396\end{array}$ & $\begin{array}{r}221815 \\
7985\end{array}$ & $\begin{array}{r}107792 \\
2214\end{array}$ & & & & \\
\hline
\end{tabular}

Estimates based on normal distribution of subjective difference $d$ :

$\begin{array}{lrlllllll}z_{1} & -1.000 & 0.117 & 0.739 & 1.227 & 3.80 \mathrm{E}-2 & .730 & -.629 & .361 \\ z_{2} & 1.000 & 1.861 & 2.410 & 2.846 & 2.48 \mathrm{E}-2 & .609 & -.425 & .199 \\ (D / T)^{*} & 0.000 & 1.134 & 1.885 & 2.515 & 1.91 \mathrm{E}-2 & .830 & -.504 & .262 \\ \left(\sigma_{c} / T\right)^{*} & 1.000 & 1.147 & 1.197 & 1.235 & 1.05 \mathrm{E}-1 & .076 & -.109 & .085 \\ \left(D / \sigma_{c}\right)^{*} & 0.000 & 0.989 & 1.575 & 2.036 & 3.18 \mathrm{E}-2 & .669 & -.528 & .278\end{array}$

Estimates based on logistic distribution of subjective difference $d$ :

$\begin{array}{lrlllllll}\ell_{1} & -0.919 & 0.103 & 0.667 & 1.153 & 3.32 \mathrm{E}-2 & .678 & -.536 & .380 \\ \ell_{2} & 0.920 & 1.891 & 2.659 & 3.369 & 5.46 \mathrm{E}-3 & .812 & -.261 & .145 \\ (D / T)^{*} & 0.000 & 1.116 & 1.670 & 2.040 & 6.16 \mathrm{E}-2 & .667 & -.746 & .378 \\ \left(\sigma_{c} / T\right)^{*} & 1.087 & 1.119 & 1.004 & 0.902 & 2.04 \mathrm{E}-1 & -.067 & -.134 & .160 \\ \left(D / \sigma_{c}\right)^{*} & 0.000 & 0.997 & 1.663 & 2.261 & 1.54 \mathrm{E}-2 & .745 & -.399 & .263\end{array}$

Estimates based on $t(4)$ distribution of subjective difference $d$ :

\begin{tabular}{lrllllrrr}
$t_{1}$ & -0.807 & 0.088 & 0.578 & 1.027 & $3.04 \mathrm{E}-2$ & .599 & -.446 & .364 \\
$t_{2}$ & 0.807 & 1.809 & 2.834 & 4.075 & $2.75 \mathrm{E}-3$ & 1.083 & .239 & .193 \\
$(D / T)^{*}$ & 0.000 & 1.103 & 1.512 & 1.674 & $1.36 \mathrm{E}-1$ & .543 & -.941 & .447 \\
$\left(\sigma_{c} / T\right)^{*}$ & 1.239 & 1.162 & 0.886 & 0.656 & $4.14 \mathrm{E}-2$ & -.202 & -.153 & .245 \\
$\left(D / \sigma_{c}\right)^{*}$ & 0.000 & 0.949 & 1.706 & 2.551 & $1.87 \mathrm{E}-3$ & .841 & -.104 & .280 \\
\hline
\end{tabular}


(a positive quadratic trend for $[D / T]^{*}$ and a positive linear trend for $\left.\left[\sigma_{c} / T\right]^{*}\right)$. Just as for the empirical data, the use of $t(4)$ quite efficiently counteracts this effect by stabilizing the deviate difference. Using logits has a similar but smaller effect.

Nonzero $\sigma_{T}$ has an effect opposite to that of nonzero $\sigma_{\sigma}$ (due to its increasing the number of "equal" judgments), and also more than outweighs the effect of $\sigma_{\sigma}$ on $(D / T)^{*}$ when both factors are introduced $\left(\sigma_{T}=\sigma_{\sigma}=0.8\right.$, Table 3D). In these situations, to use logits or $t(4)$ has a detrimental effect on the estimates.

The $z$, logit, and $t(4)$ values from Table $3 \mathrm{C}\left(\sigma_{T}=0\right.$, $\left.\sigma_{\sigma}=0.8\right)$ are plotted in Figures 3-5 for comparison with the results from Hellström (1977a). (The three lowermost triangles in each figure are based on additional simulations for $D=-1,-2$, and -3 .) In each figure, the general trend of the empirical values (circles) is recovered by the simulated values (triangles). A slight systematic deviation indicates that the "true" empirical $\sigma_{c}$ values are generally somewhat lower than the value 1 , which was used in the simulation (cf. Table 2).

\section{GENERAL DISCUSSION}

The present study does not purport to "solve" the nonnormality problem either theoretically or practically, just to point out its existence and suggest some explanations and remedies.

The Monte Carlo study explored very few and perhaps overschematized cases out of the infinitely many possibilities. However, it showed that interindividual variability of $\sigma_{d}$ has important consequences for the pooled distribution of $d$, and thus also for estimating its mean and standard deviation from three-category stimulus comparisons. A nonzero $\sigma_{\sigma}$ causes an inflation of these estimates in units of the subjective "equal" category half-width $T$; this effect strikingly resembles the empirical effect in the duration and loudness comparison data of Hellström (1977a, $1977 \mathrm{~b}, 1978,1979)$. Because there are also theoretical reasons to expect a nonzero $\sigma_{\sigma}$, we may conclude that this is one likely factor behind the inflation effect, and that $\sigma_{T}$ is comparatively unimportant. (The interindividual variation in the tendency to judge two stimuli as "equal" may at least partly result from variation in $\sigma_{d}$ rather than in $T$.) Although modeling the $d$ distribution with the logistic distribution in lieu of the ND leads to slight improvements in estimation, the $t$ distribution with a small $d f$ seems better suited (but not necessarily the best choice).

The nonnormality problem is probably the most severe with pooled group data. However, in individual data, the $S D$ of the subjective quantity may vary over trials if the observer's attention level fluctuates, yielding a total distribution heavier tailed than the ND. A realistic model of this distribution should be particularly important when one uses Equation 3 or the method of successive categories, but also in, for example, studies using the con- stant methods (Figures 1-2) or signal detection methodology (cf. Luce, 1959), where nonlinear ROC curves in $z$-coordinates may be due to nonnormality.

\section{REFERENCES}

Abramowttz, M., \& Stegun, I. A. (EDs.) (1965). Handbook of marhematical functions. New York: Dover.

BERKSON, J. (1944). Applications of the logistic function in bioassay. Joumal of the American Statistical Association, 39, 357-365.

BERKSON, J. (1953). A statistically precise and relatively simple method of estimating the bioassay with quantal response, based on the logistic function. Joumal of the American Statistical Association, 48, 565-599.

BorING, E. G. (1950). A history of experimental psychology (2nd ed.). New York: Appleton-Century-Crofts.

Burke, C. J., \& Zinnes, J. L. (1965). A paired comparison of paired comparisons. Joumal of Mathematical Psychology, 2, 53-76.

Bush, R. R. (1963). Estimation and evaluation. In P. Suppes, L. Zinnes, R. R. Bush, \& E. Galanter (Eds.), Handbook of mathematical psychology (Vol. 1, pp. 429-469). New York: Wiley.

Chandler, J. P. (1969). STEPIT-finds local minima of a smooth function of several parameters. Behavioral Science, 14, 81-82 (CPA 312).

Cramér, M. (1946). Mathematical methods of statistics. Princeton, NJ: Princeton Mathematical Series.

EISLER, H. (1965). The connection between magnitude and discrimination scales and direct and indirect scaling methods. Psychometrika, 30, $271-289$.

EKMAN, G. (1956). Discriminal sensitivity on the subjective continuum Acta Psychologica, 12, 233-243.

EKMAN, G. (1959). Weber's law and related functions. Journal of Psychology, 47, 343-352.

Fechner, G. T. (1860). Elemente der Psychophysik [Elements of psychophysics]. Leipzig: Breitkopf \& Härtel.

FEINBERG, S. (1980). The analysis of cross-classified data (2nd ed.). Cambridge, MA: MIT Press.

Galton, F. (1869). Hereditary genius. London: Macmillan.

GlenN, W. A., \& DAvid, H. A. (1960). Ties in paired-comparison judgments using a modified Thurstone-Mosteller model. Biometrics, 16, 86-109.

GreENBerG, M. G. (1965). A modification of Thurstone's law of comparative judgment to accomodate a judgment category of "equal" or "no difference." Psychological Bulletin, 64, 108-112.

Guilford, J. P. (1954). Psychometric methods. New York: McGraw-Hill.

Hellström, $\dot{A}$. (1977a). Differential sensation weighting as the basic cause of time-errors (Report No. 498). Stockholm: University of Stockholm, Department of Psychology.

Hellström, A. (1977b). Time errors are perceptual. Psychological Research, 39, 345-388.

Hellström, Ä. (1978). Factors producing and factors not producing time errors: An experiment with loudness comparisons. Perception \& Psychophysics, 23, 433-444.

Hellström, $\dot{A}$. (1979). Time errors and differential sensation weighting. Joumal of Experimental Psychology: Human Perception \& Performance, $5,460-477$.

Hellström, $\dot{A}$. (1985). The time-order error and its relatives: Mirrors of cognitive processes in comparing. Psychological Bulletin, 97, 35-61.

Hellström, $\dot{A}$. (1989). What happens when we compare two stimuli? In S. Dornic \& G. Ljunggren (Eds.), Psychophysics in action (pp. 25-39). Berlin: Springer-Verlag.

Hoaglin, D. C., Mosteller, F., \&ukey, J. W. (1983). Understanding robust and exploratory data analysis. New York: Wiley.

Johnson, N. L., KoTZ, S. (1970). Univariate continuous distributions (Vol. 2). Boston: Houghton Mifflin.

LuCE, R. D. (1959). Individual choice behavior. New York: Wiley. Luce, R. D., \& Galanter, E. (1963). Discrimination. In P. Suppes, 
L. Zinnes, R. R. Bush, \& E. Galanter (Eds.), Handbook of mathematical psychology (Vol. 1, pp. 191-243). New York: Wiley.

Olson, C. L., \& OGILviE, J. C. (1972). The method of constant stimuli with two or more categories of response. Joumal of Mathematical Psychology, 9, 320-340.

Petrusic, W. M. (1981, June). Explicit counting in duration discrimination. Paper presented at the relational judgment symposium at the meeting of the Canadian Psychological Association, Toronto.

RAsCH, G. (1960). Probabilistic models for some intelligence and attainment tests. Copenhagen: Nielsen \& Lydiche.

SaITo, T. (1988). Psychophysical scaling of the asymmetry in comparative judgments (Hokkaido Behavioral Science Report Series M, No. 12). Sapporo, Japan: Hokkaido University, Bungakubu, Department of Behavioral Science.

SJö̈ERG, L. (1967). Successive intervals scaling of paired comparisons. Psychometrika, 32, 297-308.

Stevens, S. S. (1957). On the psychophysical law. Psychological Review, 64, 153-181.

Subroviak, M. J. (1974). Remarks on the method of paired comparisons: The effect of non-normality on Thurstone's comparative judgment model. Educational \& Psychological Measurement, 34, 829-835.

Thurstone, L. L. (1927a). A law of comparative judgment. Psychological Review, 34, 273-286.

Thurstone, L. L. (1927b). Psychophysical analysis. American Journal of Psychology, 38, 368-389.

TORGERSON, W. S. (1958). Theory and methods of scaling. New York: Wiley.

Tukey, J. W., \& Mclaughun, D. H. (1963). Less vulnerable confidence and significance procedures for location based on a single sample: Trimming/winsorization 1. Sankhya: The Indian Journal of Statistics (Series A), 25, 331-352.
Watson, C. S., Kellogg, S. C., Kawanisk, D. T., \& Lukas, P. A. (1973). The uncertain response in detection-oriented psychophysics. Journal of Experimental Psychology, 99, 180-185.

WoOdWorth, R. S., \& SChlosBeRG, H. (1954). Experimental psychology (rev. ed.). New York: Holt.

\section{NOTES}

1. In Hellström (1977a, 1977b, 1978, 1979), the symbol $t$ was used. The change to $\tau$ is made to avoid confusion with the deviates of the $t$ distribution.

2. The individual response distributions are given in Hellström (1979, Appendix, Table A). Ten errors were noted in this table long after its publication. Corrections follow (group, pair, judgment category: correct figure [wrong figure]): 1, 1, 1:21 (22); 2, 6, 1:14 (13); 3, 7, 2:15 (16); $7,15,1: 44(43) ; 8,7,1: 19(20) ; 10,6,2: 36(35) ; 11,5,2: 60$ (61); 15, 10, 2:36 (35); 16, 13, 2:74 (73); 16, 15, 2:40 (41). These errors have an entirely negligible influence on the results. All computations in the present article are based on the correct entries.

3. This arrangement is equivalent to creating a correlation over subjects of -1 between the category limits $\tau_{1}$ and $\tau_{2}$,. Any other value of this correlation would have yielded not only a variability in $T=$ $\left(\tau_{1}-\tau_{2}\right) / 2$, but also a variability in the category position or "bias" = $\left(\tau_{1}+\tau_{2}\right) / 2$. Introducing the latter would be indistinguishable from adding interindividual variability to $d$. The present design is thus the most clear-cut way of introducing variability in $T$ to study its effect on the $c$ distribution.

(Manuscript received April 18, 1991; revision accepted for publication December 31, 1992.) 